\title{
BUSCAMIN: UM APLICATIVO DE BUSCA MINERAL
}

\author{
M. R. S. ALMEIDA* e L. D. A. SOUSA \\ Instituto Federal de Educação, Ciência e Tecnologia do Amapá - IFAP \\ rafaelmeida@outlook.com*
}

Artigo submetido em outubro/2015 e aceito em abril/2016

DOI: $10.15628 /$ holos.2016.3517

\section{RESUMO}

O conhecimento de mineralogia atualmente é bem desenvolvido, porém muitas vezes aplicado de forma equivocada, sem fundamento. Sabe-se que a tarefa de identificação mineral não é simples e envolve muitos fatores que só pessoas com experiência conseguem reconhecer e associar a cada rocha que se deseja caracteriza; com isso o aplicativo BuscaMin é um projeto de difusão dos conhecimentos de mineralogia através de uma ferramenta para ser utilizada em dispositivos móveis. Com esse aplicativo o usuário é capaz de identificar uma grande variedade de minerais, desde os mais conhecidos, até os menos comuns, visto que estão cadastrados mais de 70 minerais nesse programa. Essa ferramenta está organizada em seis pastas contendo os minerais que possuem composições químicas semelhantes agrupadas. As opções disponibilizam listas de minerais, que ao serem selecionados revelam a cor, cor do traço, dureza, densidade, brilho, clivagem, fratura e utilização de cada mineral, bem como uma foto da respectiva formação rochosa. As buscas podem ser feitas por qualquer pessoa, bastando está conectada à internet com um dispositivo que contenha sistema Android ou IOS.

PALAVRAS-CHAVE: Mineralogia, identificação mineral, aplicativo, minerais.

\section{BUSCAMIN: A MINERAL SEARCH APPLICATION}

\begin{abstract}
Knowledge of mineralogy is now well developed, but often applied wrongly, unfounded. It is known that mineral identification task is not simple and involves many factors that only people with experience can recognize and associate with each rock that is desired features; thus the BuscaMin application is a broadcasting project of the mineralogy of knowledge through a tool to be used on mobile devices. With this application the user is able to identify a wide range of minerals, from the known to the less common, as they are registered more
\end{abstract}

than 70 minerals in this program. This tool is organized into six folders containing minerals that have similar chemical compositions grouped. The options offer lists of minerals, which are selected to reveal the color, stroke color, hardness, density, brightness, cleavage fracture and use of each mineral, as well as a photo of the respective rock formation. Searches can be made by anyone, simply is connected to the internet with a device that contains Android or IOS system.

KEYWORDS: Minerology, mineral identification, application, minerals. 


\section{INTRODUÇÃO}

Aplicativos são ferramentas tecnológicas constituídas para diversas finalidades, porém sempre com o objetivo de facilitar um processo, uma busca, uma comunicação. Os aplicativos existem há pelo menos 10 anos, contudo se tornaram populares a partir de 2007 com o aumento de consumo dos "smartphones", visto que para utilizar as funcionalidades oferecidas pelos por esses programas é necessário estar conectado a um dispositivo móvel com sistema IOS ou Android (MEIER, 2012) (DIKKERS et al., 2011).

Dentre os campos de estudo da mineração, a mineralogia é tida como umas das principais beneficiadas pelo desenvolvimento tecnológico, onde a criação de um aplicativo que facilite a identificação de minerais seria exemplo de como aliar tecnologia e mineração em pro dos estudantes e profissionais da área (PANCHLER et al., 2009).

Essa nova ferramenta possibilitará a identificação mais rápida dos principais minerais conhecidos, simplificando o processo de pesquisa e aprendizagem de profissionais e alunos da área mineral, mais especificamente da mineralogia, pois não são poucas as vezes que se têm dúvidas quanto ao nome e propriedades dos minerais, devendo assim recorrer a elementos que tragam suporte para esclarecer os questionamentos sobre qual elemento está se tratando (KLOPFER, 2012).

A fim de diminuir os erros de identificação mineral e melhorar a qualidade das pesquisas e desenvolvimento dos trabalhos, esse aplicativo traz maior segurança no momento de afirmar qual o tipo de mineral tem maior possibilidade de ser o que está vendo, oferecendo indícios práticos como fotos e propriedades dos minerais para embasar a suspeita. Além de pessoas ligadas a mineração, qualquer membro da comunidade poderá utilizar o aplicativo com o intuito de esclarecer dúvidas quanto à origem dos materiais que possui, evitando assim ser vítima de prováveis enganos quanto ao tipo de mineral que consumiu ou vendeu, já que é um aplicativo de fácil utilização e acessível a todos (CARABALLO, 2015).

A mineração envolve um campo extenso de áreas que se complementam dando suporte para entender o objetivo geral dessa ciência, dentre esse campo se encontra a mineralogia, caracterizada como a ciência que estuda os minerais, identificando suas propriedades para então denominá-los, atribuindo a eles valor econômico e científico. Dar nomes aos elementos da natureza não é uma tarefa fácil, pois são muitas as possibilidades de combinações de letras que possuam significado lógico e corretamente relacionado com o objeto que está se classificando, diante disso são geradas normas para a correta denominação dos elementos, especificamente dos minerais (NICKEL, 1987) (BAILEY, 1981).

A partir da identificação de um mineral é possível conhecê-lo e torná-lo identificável por uma gama maior de pessoas, já que seu nome será capaz de indicar a qualquer indivíduo de que mineral está se tratando e quais as propriedades que ele carrega consigo. Segundo o International Mineralogical Association os nomes dos minerais remetem ao valor que ele possui, tomando o ouro como exemplo, no momento em que uma rocha passa a ser chamada de ouro, o seu significado muda, já que ela se torna mais valiosa e com características apreciadas por muitos (MANDARITO et al., 1984). 
Devem-se sempre pensar em meios menos difíceis de atingir objetivos, ou seja, formas práticas, rápidas e consistentes de se obter o que se quer naquele momento; com isso a tecnologia é a principal aliada do homem no que diz respeito à facilidade, que depende de veículos cada vez menos rebuscados para funcionar de maneira plena e satisfatória. A aliança da mineração à inovação garante que seus portadores carregassem consigo um guia prático ilustrado com os principais minerais conhecidos, sendo capaz de ter em mãos um verdadeiro livro de mineralogia, mais adaptado para trabalhos em campo, e que possibilita uma busca rápida e avançada sobre o material que se procura (CALÓGERAS, 1904).

A mineração uma é das áreas de maior destaque no cenário mundial, pelo fato dela tornar possível grande parte dos serviços que a humanidade desfruta atualmente, assim, fazer investimentos para esse ramo é sem dúvida algo que trará benefícios para vários segmentos da sociedade. Em vista disso, extrair as funcionalidades das tecnologias a serviço da mineração é um fator crucial para o bom andamento da sociedade em geral, não apenas para as pessoas diretamente envolvidas. Os aplicativos são a melhor forma de concretizar os bons exemplos que os avanços tecnológicos podem trazer para os mais variados segmentos, pois eles podem ser editados da maneira que se queria e como se deseja que ele funcione, assim construindo uma ferramenta sólida para desempenhar funções bem específicas que atendam a demanda do seu gerador (SOUZA, 2011) (PANCHLER et al., 2009).

Dessa forma, objetivo do trabalho é a criação de um aplicativo que facilite a identificação mineral, divulgando os conhecimentos mineralógicos e diminuindo os erros na hora de identificar uma formação rochosa. Para isso utilizam-se ferramentas bibliográficas e tecnológicas na sua construção, dando fidedignidade aos dados nele contido, e visando facilitar as buscas dos elementos dos minerais, bem como seu processo de fixação pelo usuário. Assim, os interessados poderão contar com uma ferramenta sólida e segura nos momentos de dúvidas quanto as peculiaridades dos minerais.

\section{METODOLOGIA}

Para o desenvolvimento do aplicativo denominado "BuscaMin" será utilizado o website Fábrica de Aplicativos, que se trata de um site gratuito voltado para a criação de aplicativos inéditos que atenda às necessidades do seu criador, totalmente moldado pelo idealizador para atender as necessidades previamente estabelecidas.

O site disponibiliza três campos principais, que são: design, conteúdo e perfil. Onde o campo design trata das cartelas de cores, designers e formas que o aplicativo possuirá; já o conteúdo é a principal área para anexar assuntos em textos, fotos, vídeos, notas e outros; por fim o campo perfil salva o "app", dando a ele uma definição, com imagem ícone e tornando-o disponível para aqueles que desejarem baixar e utilizar a nova ferramenta.

Como foi dito, o conteúdo é o que de fato garante individualidade e a finalidade para qual o aplicativo foi criado, logo para a sua definição é necessária uma fonte de dados confiável, que seja cientificamente aceita pelo público acadêmico, em vista disso serão utilizados como referências os livros "Guia dos Minerais" (2008) do autor Walter Schumann e "Manual de Ciência dos Mineirais" (2012) dos autores Klein \& Dutrow, como fontes principais dos dados contidos no aplicativo. 
Logo os textos, imagens, informações e propriedades dos minerais contidas no aplicativo serão retiradas das fontes supracitada, assim garantido que identificações minerais equivocadas sejam evitadas.

O aplicativo contará com campos para serem escolhidos pelo usuário, os quais são: elementos nativos, sulfetos e sulfossias, minerais halogêneos, óxido/hidróxidos, nitratos/carbonatos, sulfatos/fosfatos, silicatos, boratos, tungstatos, arsenatos/vanadatos; assim o usuário poderá selecionar a opção de busca no aplicativo, onde automaticamente todos os minerais que estiverem cadastrados na aba escolhida serão apresentados para análise individual de quem está procurando, cabendo ao mesmo escolher qual a melhor opção se enquadra na sua busca.

Caso a busca não apresente resultados ou apresente apenas um resulta significa que na base de dados escolhida como fonte do "app" havia apenas um mineral com as características desejadas ou não havia nem um relacionado com as buscas.

\section{RESULTADOS E DISCURSSÕES}

O aplicativo BuscaMin é uma ferramenta auxiliadoras das buscas em mineralogia, onde nele estão contidos 84 minerais cadastrados, cada um contendo foto e texto que descreve: a cor, cor do traço, dureza, densidade, brilho, clivagem, fratura e utilização. Os minerais foram divididos em dez classes, como já citados no item anterior. Os quais foram agrupados devidos suas similaridades, principalmente no que diz respeito à origem e composição química.

Esse app reuniu os principais minerais já catalogados e descritos, onde na aba de elementos nativos é possível encontrar descritos os minerais de ouro, prata, cobre, diamante, grafita e enxofre; todos mundialmente populares, com aplicações bem definas e conhecidas há bastante tempo. Já na opção de sulfetos se encontram os minerais que possuem enxofre em sua composição química, que são: pirita, marcassita, cinábrio, antimonita, calcopirita, galena, realgar, arsenopirita, pirita-magnética e esfarelita, os quais se encontram devidamente descritos.

Em seguida se têm os minerais halogêneos, composto pela halita e flourita, ambos muito comuns e empregados como matéria-prima na indústria química. Já na opção de óxido/hidróxidos estão contidos os minerais que dispõe em sua fórmula química as mais diversas ligações possíveis entre oxigênio e outros elementos, e hidrogênio com outros elementos, e são: corindon, magnetita, goethita, hematita, psilomelano, cassiterita, cristal de rocha, ametista, ágata, madeira fossilizada, olho de tigre e opala; onde a maioria é utilizada para a confecção de adornos e produtos ornamentais.

$\mathrm{Na}$ aba de nitratos/carbonatos estão disponíveis para consulta os minerais: calcita, rodocrosita, dolomita, aragonita, siderita, smithsonita, azurita e malaquita, pois todos são frutos de ligações entre o nitrogênio e outros elementos, e o carbono associado a outros elementos sob forma de $\mathrm{CO}_{3}$

Ainda se tem a opção de sulfatos/fosfatos, onde se têm os minerais: celestita, barita, gipsita, rosa do deserto, piromorfita, wavellita, turquesa e apatita. Dentro da opção silicatos podese encontra: o berilo, a cianita, o epídoto, a rodonita, o lápis-lazúli, a moscovita, o ortoclásio, o plagioclásio, a crisocola, o zircão, a olivina, o topázio, a augita, a hornblenda, o actonolita, o 
crisotilo, a estaurolita, a almandina, a jadeíta, o dioptásio, a sodalita, o espodumênio, a hemimorfita, a turmalina, o talco e a natrolita.

$\mathrm{Na}$ aba da opção boratos, é possível consultar as propriedades físicas dos minerais bórax, kernita, ulexita e colemanita. Bem como, ao abrir a opção dos tungstatos encontra-se os minerais volframita, scheelita e wulfenita. Por fim, estão disponíveis informações sobre os minerais vanadinita, eritrita, ambligonita, autunita e canotita na aba dos arsenatos e vanadatos.

Dessa forma há uma vasta quantidade de informação relevante para ser consultada no BuscaMin, de forma simples e direta. É possível tirar dúvidas, resgatar informações, fazer buscas rápidas, aprender, estudar, e outras funcionalidades oferecidas por esse programa para dispositivos móveis.

Agora os estudantes da área mineral e a comunidade em geral contam com um aplicativo totalmente original que traz informações confiáveis a respeito dos minerais, podendo ser consultada em qualquer lugar em celulares com sistema Android ou IOS, bastando está conectado a Internet para ter em mãos informações úteis capazes de auxiliar significativamente as pesquisas.

O aplicativo BuscaMin está disponível em: http://app.vc/buscamin, podendo ser acessado por celulares ou computadores conectados à Internet. Para baixar o basta digitar o endereço na URL do navegador do "smartphone", onde surgirá a opção de download, que ao ser selecionada iniciará automaticamente a instalação do aplicativo no celular.

$\mathrm{Na}$ interface de acesso inicial observa-se uma lista de com as opções: Elementos Nativos, Sulfetos e Sulfossias, Minerais Halogêneos, Óxido/Hidróxidos, Nitratos/Carbonatos, Sulfatos/Fosfatos, Silicatos, Boratos, Tungstatos, Arsenatos/Vanadatos, como se vê no Apêndice A; para acessar as informações basta clicar na opção desejada que automaticamente carregarão todos os minerais pertencentes a lista selecionada. Após isso o usuário pode definir o mineral de interesse e consultar as informações cadastradas e fotos.

Para a melhor utilização sugere-se que o usuário esteja tendo contato com o mineral pesquisado para consegui entender melhor as características desse mineral, pois elementos como cor, cor do traço, dureza, densidade, brilho, clivagem e fratura só podem ser definidos com a posse do mineral; logo para facilitar a relação entre as propriedades presentes no aplicativo e as verdadeiras características do mineral que está se buscando a observação prática se torna fundamental. Se o usuário está pesquisando por interesse nos minerais contidos no aplicativo, é dispensável a existência do mineral em mãos.

Na Figura 1 observamos a interface de entrada do Buscamin, a qual apresenta as opções gerais disponíveis ao usuário. 


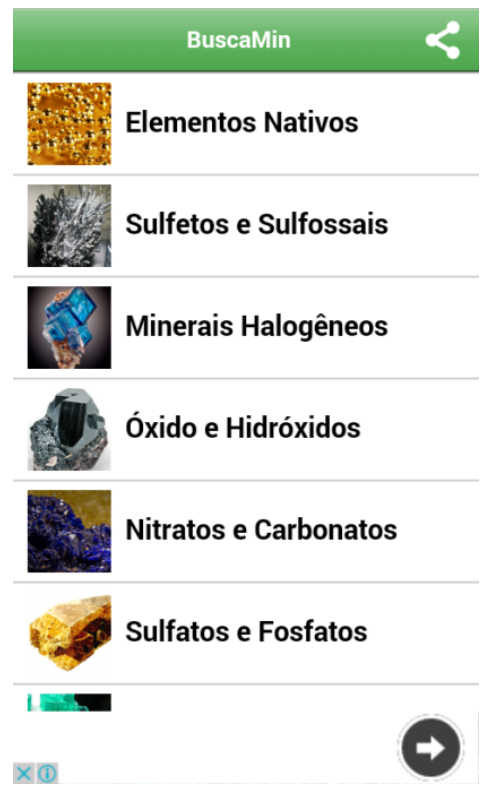

Figura 1: Interface de entrada do aplicativo Buscamin.

Dando prosseguimento, ao selecionar uma das opções da interface inicial, abrirá a lista de minerais correspondentes ao grupo, como vemos na Figura 2, onde a opção de minerais elementos está sendo exemplificada como seção selecionada.

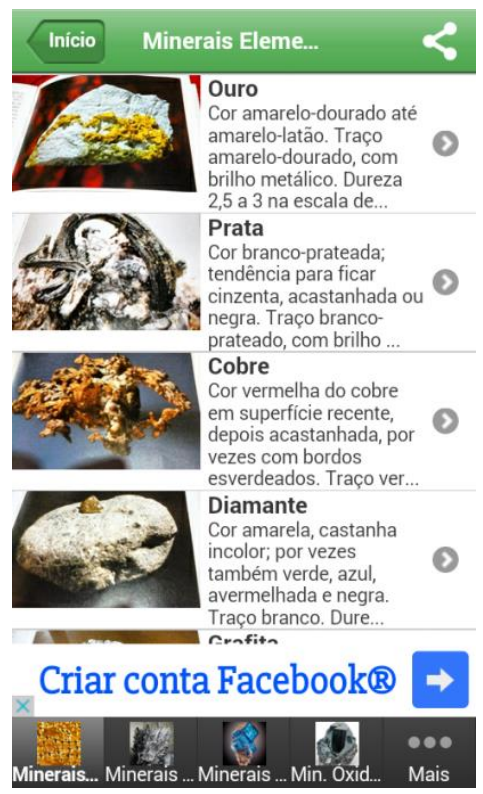

Figura 2: Opção de minerais elementos selecionada.

Para concluir a busca, e acessar as informações exclusivas do mineral de interesse, é só clicar no nome da opção que se deseja, e esperar alguns segundos enquanto os dados são gerados. A Figura 3 mostra a opção ouro selecionada, como produto de uma busca finalizada. 


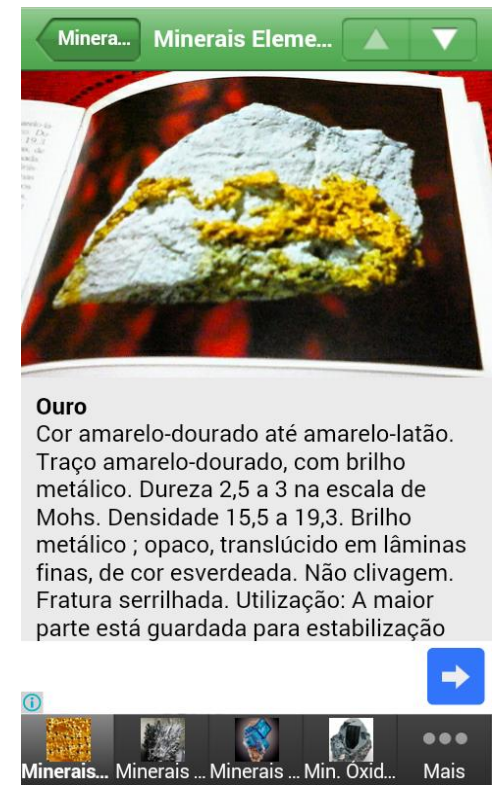

Figura 3: Interface de resultado final da busca, na opção ouro.

Assim vemos que o uso correto do aplicativo BuscaMin é simples e disponível para todos, não exigindo habilidades técnicas ou conhecimentos específicos para sua plena utilização.

Chama-se a atenção para o fato do BuscaMin ter sido desenvolvido para facilitar a identificação mineral, não podendo ser utilizado como única ferramenta a ser consultada.

\section{CONCLUSÃO}

A mineralogia representa um campo amplo de estudos da mineração, sempre com o objetivo de entender como essas formações rochosas naturais se desenvolvem durante anos, resultando em produtos belos e apreciados por todos. $\mathrm{O}$ aplicativo BuscaMin é um novo produto pronto para o uso de todos, trazendo consigo inúmeros benefícios como: difusão dos conhecimentos de mineralogia, diminuição dos erros de identificação mineral, facilita o processo de aprendizagem, redução do tempo de consulta de informações minerais, entre outros. Vale lembrar que esse aplicativo é capaz de oferecer informações seguras sobre minerais que se têm dúvidas, contudo nunca se de afirmar que uma rocha é o mineral que se pensa apenas com as informações nele contidas, visto que ele é limitado a oferecer informações práticas de resultados de testes, mas nem sempre os testes obtêm resultados iguais, dependendo da região que se encontra e da ocorrência mineralógica.

Sendo assim, espera-se que o público desfrute de todas as utilidades disponibilizadas pelo BuscaMin, pois esse aplicativo é capaz de cessar questionamentos em tempo real; podendo ser procurado a qualquer hora do dia, dando a liberdade aos interessados acessarem as informações como acharem mais produtivo, e empregá-las aonde julguem ter maior finalidade. Contudo, não se deve esquecer que os livros são valiosos e o conhecimento inesgotável; logo o ideal é sempre recorrer a diferentes fontes para se ter certeza da informação colhida, e o BuscaMin atribui-se como um desses difusores do saber. 


\section{REFERÊNCIAS BIBLIOGRÁFICAS}

1. ADUSUMILLI M.S., KIEFT C., BURKE E.A.J. Tantal-aeschynite, a new mineral of the aeschynite group from the Borborema Region, north-eastern Brazil. Mineralogical Magazine, 39:571576, 1974.

2. BAILEY, S.W. Nomenclature for regular intentratifications. CanMineral., 19:651-655, 1981.

3. CALÓGERAS, J.P. As minas do Brasil e sua legislação. Rio de Janeiro: Imprensa Nacional, v. I, 477p, 1904.

4. CARABALLO M.A., MICHEL F.M., HOCHELLA JR. M.F. The rapid expansion of environmental mineralogy in unconventional ways: Beyond the accepted definition of a mineral, the latest technology, and using nature as our guide. American Mineralogist, 100:14-25, 2015.

5. DIKKERS, S.; MARTIN, J.; COULTER, B. Mobile Media Learning: Amazing uses of mobile devices for learning. USA: Etcpress, 2012.

6. KLEIN \& DUTROW. Manual de ciências minerais; tradução de revisão técnica: Rualdo Menegat.- 23 ed.-Porto Alegre: Bookman, 2012.

7. KLOPFER, E. Augmented Learning: Research and design of mobile educational games. USA: MIT Press, 2008.

8. MANDARINO, J.A.; NICKEL, E.H.; CESBRON, F. Rules of procedure of the Comission on New Minerals and Mineral Names. International Mineralogical Association. Am. Mineral., 59:563564, 1984.

9. MATTAR, J. Games em Educação: Como os nativos digitais aprendem. São Paulo: Person, 2009.

10. MEIER, R. Professional Android Application Development. John Wiley \& Sons, 2012.

11. NICKEL E.H. \& GRICE J.D. The IMA Commission on New Minerals and Mineral Names: procedures and guidelines on mineral nomenclature. Canadian Mineralogist, 36:913-926, 1998.

12. NICKEL E.H. The definition of a mineral. Montreal: Can. Miner, 1995.

13. NICKEL E.H., MANDARINO J.A. Procedures involving the IMA Commission on New Minerals and Mineral Names and guidelines on mineral nomenclature. Am. Miner., 1987.

14. PACHLER, N.; BACHMAIR, B.; COOK, J.; KRESS, G. (2009) Mobile Learning: Structures, Agency, Practices. USA: Springer, 2009.

15. SCHUMANN, W. Guia dos Minerais. Barueri, SP: DISAL, 2008.

16. SOUZA, J. P. T. Projeto e Desenvolvimento de um Aplicativo de Geometria para Android. Monografia de Conclusão de Curso. São Carlos: Instituto de Ciências Matemáticas e de Computação, Universidade de São Paulo, 2011.

17. TRAXLER, J.; KUKULSKA-HULME, A. Mobile Learning in Developing Countries: The Knowledge Series. Canada: Commonwealth of Knowledge, 2005. 